\title{
A Stochastic Epidemic Model with Constant Immigration and Multi-Dimensional Noises
}

\author{
L.J. Hu, W.Q. Shen \\ Department of Applied Mathematics \\ Donghua University \\ Shanghai, China
}

\begin{abstract}
In this paper we extend a classical SIS epidemic model from a deterministic framework to a stochastic one by introducing two random perturbations in the model for transmission parameter and cure parameter, and formulate it as stochastic differential equation (SDE) for the number of infectious individuals $I(t)$. Then we prove that this SDE has a unique global positive solution and establish conditions for extinction. So it is clear that the basic reproductive number $R_{0}^{D}$ for the deterministic SIS model is larger than the basic reproductive number $R_{0}^{S}$ for the SDE model, which means for the sake of extinction of $I(t)$, the condition of stochastic SIS model may be weaker than that of the deterministic SIS model. The results are illustrated by computer simulations.
\end{abstract}

Keywords-SIS model; brownian motion; stochastic differential equations; extinction; basic reproduction number

\section{INTRODUCTION}

Epidemics are commonly modeled by using deterministic compartmental models where the population amongst whom the disease is spreading is divided into several classes, such as susceptible, infected and removed individuals. If $S(t)$ denotes the number of susceptibles and $I(t)$ the number of infecteds at time $t$, Then the deterministic SIS model [4] [6] can be described as:

$$
\left\{\begin{array}{l}
\frac{d S(t)}{d t}=\Lambda-\beta S(t) I(t)+\gamma I(t)-\mu S(t), \\
\frac{d I(t)}{d t}=\beta S(t) I(t)-(\mu+\gamma) I(t)
\end{array}\right.
$$

with $S(0)+I(0)=N$, the initial total size of the population . Here $\Lambda$ is a constant immigration of susceptible, $\mu$ is the per capita death rate of susceptible and infected, and $\gamma$ is the rate at which infected individuals become cured, so $1 / \gamma$ is the average infectious period. $\beta$ is the disease transmission coefficient, so that $\beta=\frac{\lambda \mu}{\Lambda}$, where $\lambda$ is the average number of adequate contacts of an infective per day. We consider all newborn as susceptible, and by SIS model (1) we know total size of the population is

$$
N(t)=S(t)+I(t)=\frac{\Lambda-e^{\log (\Lambda-\mu N)-\mu t}}{\mu} .
$$

If $\frac{\Lambda}{\mu} \geq N$, we have $N \leq N(t) \leq \frac{\Lambda}{\mu}$; and if $\frac{\Lambda}{\mu}<N$,we have $\frac{\Lambda}{\mu}<N(t)<N$. For the sake of simplicity, we set
$\frac{\Lambda}{\mu} \geq N$, which won't change the conclusion of discussion in the paper.

Denote the basic reproduction number of this model by $R_{0}^{D}=\beta \Lambda /[\mu(\mu+\gamma)]$, we can know that [3]:

$$
\begin{aligned}
& \text { If } R_{0}^{D} \leq 1, \lim _{t \rightarrow \infty} I(t)=0 . \\
& \text { If } R_{0}^{D}>1, \lim _{t \rightarrow \infty} I(t)=\frac{\Lambda}{\mu}\left(1-\frac{1}{R_{0}^{D}}\right) .
\end{aligned}
$$

Suppose that some stochastic environmental factors act simultaneously on each individual in the population. Gray etal [1] discusses a stochastic SIS model by introducing a random perturbation to transmission coefficient $\beta$ in the case that the total size of the population $N(t)$ is a constant. In this paper we explore the case of constant immigration $\Lambda$ and two parameter perturbations both in the transmission coefficient $\beta$ and the cure rate $\gamma$.

\section{A NEW STOCHASTIC SIS MODEL}

To establish the SDE model, we introduce two random perturbations to model (1) for the transmission parameter and the cure parameter. Assume that a single infected individual makes $\beta_{1} d t$ potentially infectious contacts with each other individual in the small time interval $[t, t+d t)$, and makes $\gamma_{1} d t$ cured patients in the same time. Moreover,

$$
\left(\begin{array}{l}
\beta_{1} d t \\
\gamma_{1} d t
\end{array}\right)=\left(\begin{array}{l}
\beta d t \\
\gamma d t
\end{array}\right)+\left(\begin{array}{ll}
\sigma_{1} & \sigma_{2} \\
\sigma_{3} & \sigma_{4}
\end{array}\right)\left(\begin{array}{l}
d B_{1}(t) \\
d B_{2}(t)
\end{array}\right)
$$

Here $B_{1}(t)$ and $B_{2}(t)$ are the increment of a standard Brownian motion [2]. So that $E\left(\beta_{1} d t\right)=\beta d t, E\left(\gamma_{1} d t\right)=\gamma d t$, $\operatorname{Cov}\left(\begin{array}{l}\beta_{1} d t \\ \gamma_{1} d t\end{array}\right)=\Sigma \Sigma^{T} d t$ where $\Sigma=\left(\begin{array}{ll}\sigma_{1} & \sigma_{2} \\ \sigma_{3} & \sigma_{4}\end{array}\right)$.

Replacing $\beta d t, \gamma d t$ in deterministic SIS model (1) by $\beta_{1} d t$ and $\gamma_{1} d t$, we get the following SDE SIS model:

$$
\left\{\begin{array}{l}
d S(t)=[\Lambda-\beta S(t) I(t)+\gamma I(t)-\mu S(t)] d t-I(t)\left(\sigma_{1} S(t)-\sigma_{3}, \sigma_{2} S(t)-\sigma_{4}\right)\left(\begin{array}{c}
d B_{1}(t) \\
d B_{2}(t)
\end{array}\right) \\
d I(t)=[\beta S(t) I(t)-(\mu+\gamma) I(t)] d t+I(t)\left(\sigma_{1} S(t)-\sigma_{3}, \sigma_{2} S(t)-\sigma_{4}\right)\left(\begin{array}{c}
d B_{1}(t) \\
d B_{2}(t)
\end{array}\right)
\end{array}\right.
$$

Note that total size of the population $N(t)=S(t)+I(t)$ is the same as in equation (2), it is sufficient to study the SDE for $I(t)$ : 


$$
\begin{aligned}
d I(t) & =I(t)[\beta(N(t)-I(t))-(\mu+\gamma)] d t \\
& +I(t)\left(\sigma_{1}(N(t)-I(t))-\sigma_{3}, \sigma_{2}(N(t)-I(t))-\sigma_{4}\right)\left(\begin{array}{c}
d B_{1}(t) \\
d B_{2}(t)
\end{array}\right)
\end{aligned}
$$

\section{EXISTENCE OF UNIQUE POSITIVE SOLUTION}

Theorem 3.1. For any given initial value $S(0), I(0) \in(0, N)$, the SDE (4) has a unique global positive solution $(S(t), I(t)) \in \mathbb{R}_{+}^{2}$ for all $t \geq 0$ with probability one, namely

$$
P\left\{(S(t), I(t)) \in \mathbb{R}_{+}^{2} \text { for all } t \geq 0\right\}=1 .
$$

Proof. Regarding equation (4) as an SDE on $\mathbb{R}^{2}$, we see that its coefficients are locally Lipschitz continuous [7]. It is known that for any given initial value $S_{0}, I_{0} \in(0, N)$, there is a unique maximal local solution $(S(t), I(t))$ on $t \in\left(0, \tau_{e}\right)$, where $\tau_{e}$ is the explosion time. Let $k_{0}>0$ be sufficiently large for $1 / k_{0} \leq S_{0}, I_{0} \leq k_{0}$. For each integer $k \geq k_{0}$, define the stopping time

$$
\tau_{k}=\inf \left\{t \in\left[0, \tau_{e}\right): \min \{S(t), I(t)\} \leq 1 / k \text { or } \max \{S(t), I(t)\} \geq k\right\},
$$

where throughout this paper we set inf $\emptyset=\infty$ (as usual, $\emptyset=$ the empty set). Clearly, $\tau_{k}$ is increasing as $k \rightarrow \infty$. Set $\tau_{\infty}=\lim _{k \rightarrow \infty} \tau_{k}$, whence $\tau_{\infty} \leq \tau_{e}$ a.s. If we can show that $\tau_{\infty}=\infty$ a.s., then $\tau_{e}=\infty$ a.s. and $S(t), I(t) \in(0, N(t))$ a.s. for all $t \geq 0$. In other words, to complete the proof all we need to show is that $\tau_{\infty}=\infty$ a.s. [2].

If this statement is false, then there is a pair of constants $T>0$ and $\epsilon \in(0,1)$ such that

$$
P\left\{\tau_{\infty} \leq T\right\}>\epsilon .
$$

Hence there is an integer $k_{1} \geq k_{0}$ such that

$$
P\left\{\tau_{k} \leq T\right\} \geq \epsilon \quad \text { for all } k \geq k_{1}
$$

and

$$
N(t):=S(t)+I(t) \leq \max \left\{N, \frac{\Lambda}{\mu}\right\}:=K
$$

Define a function $V: \mathbb{R}_{+}^{2} \rightarrow \mathbb{R}_{+}$by

$$
V(S, I)=\frac{1}{I}+I^{2}+S^{2} .
$$

By the Itô formula [2], we have, for any $t \in[0, T]$ and $k \geq k_{1}$,

$$
E V\left(S\left(t \wedge \tau_{k}\right), I\left(t \wedge \tau_{k}\right)\right)=V\left(S_{0}, I_{0}\right)+E \int_{0}^{t \wedge \tau_{k}} L V(S(s), I(s)) d s
$$

where $V: \mathbb{R}_{+}^{2} \rightarrow \mathbb{R}_{+}$is defined by

$$
\begin{aligned}
L V & =\left(-\frac{1}{I^{2}}+2 I\right)[\beta S I-(\mu+\gamma) I]+I^{2}\left[\left(\sigma_{1} S-\sigma_{3}\right)^{2}+\left(\sigma_{2} S-\sigma_{4}\right)^{2}\right]\left(\frac{1}{I^{3}}+1\right) \\
& +2 S(\Lambda-\beta S I+\gamma I-\mu S)+I^{2}\left[\left(\sigma_{1} S-\sigma_{3}\right)^{2}+\left(\sigma_{2} S-\sigma_{4}\right)^{2}\right] .
\end{aligned}
$$

It is easy to show that

$$
\begin{aligned}
L V & \leq \frac{\mu+\gamma}{I}+2 \beta S I^{2}+\left[\left(\sigma_{1}^{2}+\sigma_{2}^{2}\right) S^{2}+\sigma_{3}^{2}+\sigma_{4}^{2}\right]\left(\frac{1}{I}+I^{2}\right) \\
& +2 \Lambda S+2 \gamma S I+\left(\sigma_{1}^{2}+\sigma_{2}^{2}\right) S^{2} I^{2}+\left(\sigma_{3}^{2}+\sigma_{4}^{2}\right) I^{2} \\
& \leq C_{1}\left(\frac{1}{I}+I^{2}\right)+2 \Lambda K+2 \gamma K^{2}+\left(\sigma_{1}^{2}+\sigma_{2}^{2}\right) K^{2} S^{2} \\
& \leq C_{2} V+C_{3} .
\end{aligned}
$$

where $C_{1}=\left[(\mu+\gamma) \vee\left(2 \beta K+\sigma_{3}^{2}+\sigma_{4}^{2}\right)\right]+\left[\left(\sigma_{1}^{2}+\sigma_{2}^{2}\right) K^{2}+\sigma_{3}^{2}+\sigma_{4}^{2}\right]$,

$$
C_{2}=C_{1} \vee\left[\left(\sigma_{1}^{2}+\sigma_{2}^{2}\right) K^{2}\right], \quad C_{3}=2 \Lambda k+2 \gamma K^{2} .
$$

Substituting (9) into (7), and set $C_{4}=\max \left\{C_{2}, C_{3}\right\}$, we get

$$
\begin{aligned}
E V\left(S\left(t \wedge \tau_{k}\right), I\left(t \wedge \tau_{k}\right)\right) & \leq V\left(S_{0}, I_{0}\right)+E \int_{0}^{t \wedge \tau_{k}}\left[C_{2} V(S(s), I(s))+C_{3}\right] d s \\
& \leq V\left(S_{0}, I_{0}\right)+C_{4}\left(t \wedge \tau_{k}\right)+E \int_{0}^{t \wedge \tau_{k}}\left[C_{4} V(S(s), I(s))\right] d s \\
& \leq V\left(S_{0}, I_{0}\right)+C_{4} T+C_{4} \int_{0}^{T} E V\left(S\left(s \wedge \tau_{k}\right), I\left(s \wedge \tau_{k}\right)\right) d s
\end{aligned}
$$

The Gronwall inequality [2] yields that

$$
E V\left(S\left(t \wedge \tau_{k}\right), I\left(t \wedge \tau_{k}\right)\right) \leq\left[V\left(S_{0}, I_{0}\right)+C_{4} T\right] e^{C_{4} T}
$$

Set $\Omega_{k}=\left\{\tau_{k} \leq T\right\}$ for $k \geq k_{1}$. By (6), we have $P\left(\Omega_{k}\right) \geq \epsilon$. Note that for every $\omega \in \Omega_{k}$, there is at least one of $S\left(\tau_{k}, \omega\right), I\left(\tau_{k}, \omega\right)$ equals either $1 / k$ or $k$, and hence

$$
V\left(S\left(\tau_{k}, \omega\right), I\left(\tau_{k}, \omega\right)\right) \geq\left(2 k^{2}+\frac{1}{k}\right) .
$$

It then follows from (10) that

$$
\begin{aligned}
{\left[V\left(S_{0}, I_{0}\right)+C_{4} T\right] e^{C_{4} T} } & \geq E\left[1_{\Omega_{k}}(\omega) V\left(S\left(\tau_{k}, \omega\right), I\left(\tau_{k}, \omega\right)\right)\right] \\
& \geq\left(2 k^{2}+\frac{1}{k}\right) P\left(\Omega_{k}\right) \\
& \geq \epsilon\left(2 k^{2}+\frac{1}{k}\right) .
\end{aligned}
$$

Letting $k \rightarrow \infty$ leads to the contradiction

$$
\infty>\left[V\left(S_{0}, I_{0}\right)+C_{4} T\right] e^{C_{4} T}=\infty .
$$

So we must therefore have $\tau_{\infty}=\infty$.a.s. Hence the proof is complete.

\section{EXTINCTION}

Theorem 4.1. Set

$$
\begin{aligned}
R_{0}^{S} & :=R_{0}^{D}-\frac{\left(\sigma_{1}^{2}+\sigma_{2}^{2}\right) N^{2}}{2(\mu+\gamma)}-\frac{\sigma_{3}^{2}+\sigma_{4}^{2}}{2(\mu+\gamma)}+\frac{\left(\sigma_{1} \sigma_{3}+\sigma_{2} \sigma_{4}\right) \frac{\Lambda}{\mu}}{\mu+\gamma} \\
& =\frac{\beta \Lambda}{\mu(\mu+\gamma)}-\frac{\left(\sigma_{1}^{2}+\sigma_{2}^{2}\right) N^{2}}{2(\mu+\gamma)}-\frac{\sigma_{3}^{2}+\sigma_{4}^{2}}{2(\mu+\gamma)}+\frac{\left(\sigma_{1} \sigma_{3}+\sigma_{2} \sigma_{4}\right) \frac{\Lambda}{\mu}}{\mu+\gamma}
\end{aligned}
$$

If

$$
R_{0}^{S}<1 \quad \text { and } \quad \sigma_{1}^{2}+\sigma_{2}^{2} \leq \frac{\beta \mu}{\Lambda}
$$

then for any given initial value $I(0)=I_{0} \in(0, N)$, the solution of the SDE (5) obeys 
$\limsup _{t \rightarrow \infty} \frac{1}{t} \log (I(t)) \leq \frac{\beta \Lambda}{\mu}-\mu-\gamma-0.5\left(\sigma_{1}^{2}+\sigma_{2}^{2}\right) N^{2}-0.5\left(\sigma_{3}^{2}+\sigma_{4}^{2}\right)+\left(\sigma_{1} \sigma_{3}+\sigma_{2} \sigma_{4}\right) \frac{\Lambda}{\mu}$

$<0$ a.s.

namely, $I(t)$ tends to zero exponentially almost surely. In other words, the disease dies out with probability one.

Proof. By the Itô formula [2], we have

$$
\begin{aligned}
\log (I(t)) & =\log \left(I_{0}\right)+\int_{0}^{t} f(I(s), s) d s \\
& +\int_{0}^{t}\left(\sigma_{1}[N(s)-I(s)]-\sigma_{3}, \quad \sigma_{2}[N(s)-I(s)]-\sigma_{4}\right)\left(\begin{array}{c}
d B_{1}(s) \\
d B_{2}(s)
\end{array}\right)
\end{aligned}
$$

where $f: \mathbb{R}_{+} \times \mathbb{R} \rightarrow \mathbb{R}$ is defined by

$f(x, t)=\beta N(t)-\mu-\gamma-\beta x-0.5\left[\sigma_{1}(N(t)-x)-\sigma_{3}\right]^{2}-0.5\left[\sigma_{2}(N(t)-x)-\sigma_{4}\right]^{2}(1$

However, under condition (11), we have

$$
\begin{aligned}
f(I(s), s) & =\beta N(s)-\mu-\gamma-\beta I(s)-0.5\left(\sigma_{1}^{2}+\sigma_{2}^{2}\right)(N(s)-I(s))^{2} \\
& -0.5\left(\sigma_{3}^{2}+\sigma_{4}^{2}\right)+\left(\sigma_{1} \sigma_{3}+\sigma_{2} \sigma_{4}\right)(N(s)-I(s)) \\
& =\beta N(s)-\mu-\gamma-\left(\beta-\left(\sigma_{1}^{2}+\sigma_{2}^{2}\right) N(s)\right) I(s)-0.5\left(\sigma_{1}^{2}+\sigma_{2}^{2}\right)(N(s))^{2} \\
& -0.5\left(\sigma_{1}^{2}+\sigma_{2}^{2}\right) I(s)^{2}-0.5\left(\sigma_{3}^{2}+\sigma_{4}^{2}\right)+\left(\sigma_{1} \sigma_{3}+\sigma_{2} \sigma_{4}\right)(N(s)-I(s)) \\
& \leq \frac{\beta \Lambda}{\mu}-\mu-\gamma-0.5\left(\sigma_{1}^{2}+\sigma_{2}^{2}\right) N^{2}-0.5\left(\sigma_{3}^{2}+\sigma_{4}^{2}\right)+\left(\sigma_{1} \sigma_{3}+\sigma_{2} \sigma_{4}\right) \frac{\Lambda}{\mu}<0
\end{aligned}
$$

It then follows from (13) that

$$
\begin{aligned}
\log (I(t)) & \leq \log \left(I_{0}\right)+\left(\frac{\beta \Lambda}{\mu}-\mu-\gamma-0.5\left(\sigma_{1}^{2}+\sigma_{2}^{2}\right) N^{2}-0.5\left(\sigma_{3}^{2}+\sigma_{4}^{2}\right)+\left(\sigma_{1} \sigma_{3}+\sigma_{2} \sigma_{4}\right) \frac{\Lambda}{\mu}\right) t \\
& +\int_{0}^{t}\left(\sigma_{1}[N(s)-I(s)]-\sigma_{3}, \quad \sigma_{2}[N(s)-I(s)]-\sigma_{4}\right)\left(\begin{array}{c}
d B_{1}(s) \\
d B_{2}(s)
\end{array}\right) .
\end{aligned}
$$

This implies

$$
\begin{aligned}
& \limsup _{t \rightarrow \infty} \frac{1}{t} \log (I(t)) \\
& \leq \frac{\beta \Lambda}{\mu}-\mu-\gamma-0.5\left(\sigma_{1}^{2}+\sigma_{2}^{2}\right) N^{2}-0.5\left(\sigma_{3}^{2}+\sigma_{4}^{2}\right)+\left(\sigma_{1} \sigma_{3}+\sigma_{2} \sigma_{4}\right) \frac{\Lambda}{\mu} \\
& +\limsup _{t \rightarrow \infty} \frac{1}{t} \int_{0}^{t}\left(\sigma_{1}[N(s)-I(s)]-\sigma_{3} \quad \sigma_{2}[N(s)-I(s)]-\sigma_{4}\right)\left(\begin{array}{l}
d B_{1}(s) \\
d B_{2}(s)
\end{array}\right) \text { a.s. }
\end{aligned}
$$

and since

$$
\limsup _{t \rightarrow \infty} \frac{1}{t} \int_{0}^{t}\left\{\left(\sigma_{1}^{2}+\sigma_{2}^{2}\right)[N(s)-I(s)]^{2}+\left(\sigma_{1}^{2}+\sigma_{2}^{2}\right)\right\} d s<\infty
$$

by the large number theorem for martingales [2], we have

$$
\limsup _{t \rightarrow \infty} \frac{1}{t} \int_{0}^{t}\left(\sigma_{1}[N(s)-I(s)]-\sigma_{3}, \quad \sigma_{2}[N(s)-I(s)]-\sigma_{4}\right)\left(\begin{array}{c}
d B_{1}(s) \\
d B_{2}(s)
\end{array}\right)=0 .
$$

We therefore obtain the desired assertion (12) from (16).

It is useful to observe that in the classical deterministic SIS model (1), $I(t)$ tends to 0 if and only if $R_{0}^{D} \leq 1$; while in the SDE SIS model (4), $I(t)$ tends to 0 if $R_{0}^{S}<1$ and $\sigma_{1}^{2}+\sigma_{2}^{2} \leq \frac{\beta \mu}{\Lambda}$. In other words, the conditions for $I(t)$ become extinct in the SDE SIS model may be weaker than in the deterministic SIS model. The following example illustrates this result more explicitly.
Similarily, we can prove the follwing result for the case $R_{0}^{S} \leq 1$.

Theorem 4.2. If

$$
R_{0}^{S} \leq 1 \quad \text { and } \quad \sigma_{1}^{2}+\sigma_{2}^{2}>\left(\frac{\beta \mu}{\Lambda} \vee \frac{\beta\left(\beta+\sigma_{1} \sigma_{3}+\sigma_{2} \sigma_{4}\right)+\left(\sigma_{1} \sigma_{3}+\sigma_{2} \sigma_{4}\right)\left(\beta+\sigma_{1} \sigma_{3}+\sigma_{2} \sigma_{4}\right)}{\mu+\gamma}\right)
$$

then for any given initial value $I(0)=I_{0} \in(0, N)$, the solution of the SDE (5) obeys

$$
\begin{aligned}
& \limsup _{t \rightarrow \infty} \frac{1}{t} \log (I(t)) \\
& \leq-\mu-\gamma+\frac{\beta\left(\beta+\sigma_{1} \sigma_{3}+\sigma_{2} \sigma_{4}\right)}{\sigma_{1}^{2}+\sigma_{2}^{2}}+\left(\sigma_{1} \sigma_{3}+\sigma_{2} \sigma_{4}\right) \frac{\beta+\sigma_{1} \sigma_{3}+\sigma_{2} \sigma_{4}}{\sigma_{1}^{2}+\sigma_{2}^{2}}<0 \text { a.s. }
\end{aligned}
$$

namely, $I(t)$ tends to zero exponentially almost surely. In other words, the disease dies out with probability one.

Example 4.1. Throughout the paper we shall assume that the unit of time is one day and the population sizes are measured in units of 1 million. Assume that the system parameters are given by

$$
\begin{gathered}
\beta=0.5, \Lambda=2000, N=60, \mu=20, \gamma=25 \\
\sigma_{1}=0.045, \sigma_{2}=0.04, \sigma_{3}=0.03, \sigma_{4}=0.035
\end{gathered}
$$

So the SDE SIS model (5) becomes

$d I(t)=I(t)[0.5 N(t)-0.5 I(t)-45] d t$

$+I(t)(0.045[N(t)-I(t)]-0.03, \quad 0.04[N(t)-I(t)]-0.035)\left(\begin{array}{c}d B_{1}(t) \\ d B_{2}(t)\end{array}\right)$

where $N(t)=100-0.05 e^{\log 800-20 t}$.

Noting that

$R_{0}^{S}=\frac{\beta \Lambda}{\mu(\mu+\gamma)}-\frac{\left(\sigma_{1}^{2}+\sigma_{2}^{2}\right) N^{2}}{2(\mu+\gamma)}-\frac{\sigma_{3}^{2}+\sigma_{4}^{2}}{2(\mu+\gamma)}+\frac{\left(\sigma_{1} \sigma_{3}+\sigma_{2} \sigma_{4}\right) \frac{\Lambda}{\mu}}{\mu+\gamma} \approx 0.9722<1$, and $\sigma_{1}^{2}+\sigma_{2}^{2}=0.0036 \leq \frac{\beta \mu}{\Lambda}=0.005$. We can therefore conclude, by Theorem 4.1, that for any initial value $I(0)=I_{0} \in(0,60)$ the solution of (19) obeys

a.s. $\quad \limsup _{t \rightarrow \infty} \frac{1}{t} \log (I(t)) \leq-1.2511 \quad$ a.s.

That is, $I(t)$ will tend to zero exponentially with probability one.

On the other hand, the corresponding deterministic SIS model (1) becomes

$$
d I(t)=I(t)\left[50-0.025 e^{\log 800-20 t}-0.5 I(t)-45\right] d t .
$$

Since $R_{0}^{D}>1$, it is known that, for any initial value $I(0)=I_{0} \in(0,60)$,this solution has the property

$$
\lim _{t \rightarrow \infty} I(t)=\frac{\Lambda}{\mu}\left(1-\frac{1}{R_{0}^{D}}\right)=10
$$

That is, deterministic $I(t)$ will not tend to zero (see Figure 1). Namely when $I(t)$ extincts in the SDE SIS model , $I(t)$ doesn't extinct in the deterministic SIS model. 


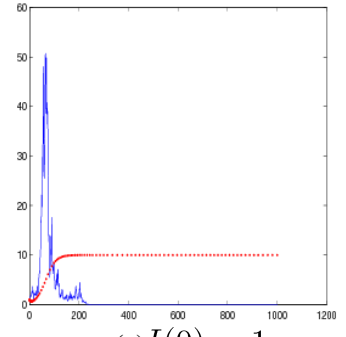

(a) $I(0)=1$

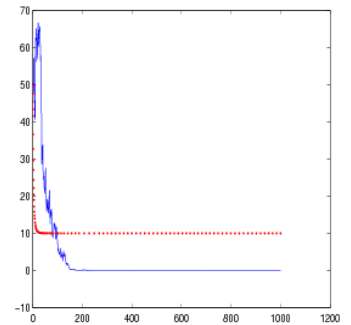

(b) $I(0)=50$
FIGURE I. SIMULATION OF EXAMPLE 4.1. THE NUMERICAL

METHOD IS EULER SCHEME WITH TIME STEP $\Delta=0.001$. THE FULL LINE DENOTES STOCHASTIC, THE DOTTED LINE DENOTES DETERMINISTIC.

Example 4.2. We keep the system parameters the same as in Example 4.1 but let $\sigma_{1}=0.095, \sigma_{2}=0.09$,so the SDE SIS model (5) becomes

$$
\begin{aligned}
& d I(t)=I(t)[0.5 N(t)-0.5 I(t)-45] d t \\
& +I(t)(0.095[N(t)-I(t)]-0.03 \quad 0.09[N(t)-I(t)]-0.035)\left(\begin{array}{c}
d B_{1}(t) \\
d B_{2}(t)
\end{array}\right)
\end{aligned}
$$

where $N(t)=100-0.05 e^{\log 800-20 t}$.

It is easy to verify that the system parameters obey condition (17). We can therefore conclude, by Theorem 4.2, that for any initial value $I(0)=I_{0} \in(0,60)$, the solution of (20) obeys

$$
\limsup _{t \rightarrow \infty} \frac{1}{t} \log (I(t)) \leq-30.049 \quad \text { a.s. }
$$

That is, $I(t)$ will tend to zero exponentially with probability one. The computer simulations shown in Figure 2 support these results clearly. We conclude that when $\sigma_{1}$ increase from 0.045 to $0.095, \sigma_{2}$ increase from 0.04 to 0.09 , it will accelerate the speed of SDE SIS model tends to 0.

\section{ACKNOWLEDGEMENTS}

The authors would like to thank the National Natural Science Foundation of China (grant no 11471071) and the Natural Science Fundation of Shanghai (grant no 14ZR1401200) for their financial support.

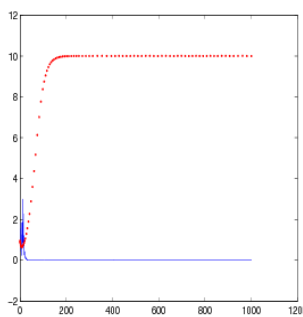

(a) $I(0)=1$

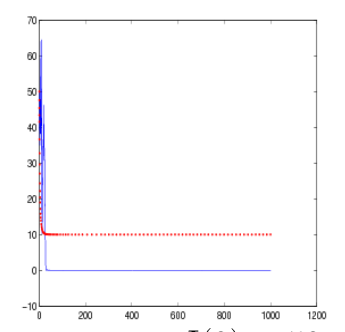

(b) $I(0)=50$
FIGURE II. SIMULATION OF EXAMPLE 4.2. THE NUMERICAL METHOD IS EULER SCHEME WITH TIME STEP $\Delta=0.001$. THE FULL LINE DENOTES STOCHASTIC, THE DOTTED LINE DENOTES DETERMINISTIC.

\section{REFERENCES}

[1] Gray, A., Greenhalgh, D., Hu, L., Mao, X. \& Pan, J., A Stochastic Differential Equation SIS Epidemic Model. SIAM J. Appl. Math.,71(3), pp. 876-902, 2011.

[2] Mao, X., Stochastic Differential Equations and Applications, 2nd Edition, Horwood: Chichester, 2007.

[3] Hethcote, H.W. \& Yorke, J.A., Gonorrhea Transmission Dynamics and Control, Lecture Notes in Biomathematics 56, Springer-Verlag, 1994.

[4] McCormack, R.K. \& Allen, L.J.S., Stochastic SIS and SIR multihost epidemic models. Proceedings of the Conference on Differential and Difference Equations and Applications, Eds. R.P. Agarwal and K. Perera, Hindawi Publishing Corporation, pp.775-786, 2006.

[5] Allen, E., Modelling with Stochastic Differential Equations, SpringerVerlag, 2007.

[6] Zhou, Y. \& Ma, Z., Global Stability of A Class of Discrete AgeStructured SIS Models with Immigration. Mathematical Biosciences And Engineering, 6(2), pp. 409-425, 2009.

[7] Mao, X. \& Yuan C., Stochastic Differential Equations with Markovian Switching, Imperial College Press, pp. 90-91, 2006. 